AROUEOLogía Y SOCIEDAd

№ 29, 2015: 9-15

1SSN: 0254-8062

RECIBIDO: ABRIL DEL 2015

ACEPTADO: MAYO DEL 2015

\title{
HOMENAJE AL DOCTOR JOSÉ MATOS MAR Y LA ANTROPOLOGÍA ANDINA
}

\author{
Alberto Bueno Mendoza \\ UnIVERSIDAD NACIONAL MAYOR DE SAN MARCOS
}

"La marcha de millones de provincianos del Otro Perú ha sido apasionante y gigantesca; salieron de sus pueblos buscando bienestar, presencia y reconocimiento en la vida nacional y, en el camino, acabaron construyendo modernidad, democracia y una nueva sociedad nacional andina. Todo ello para descubrir que la meta es promisoria, pero el tramo restante tan duro "como todo lo vivido".

José Matos Mar, 2012.

\section{Prefacio.}

El Dr. José Matos Mar (Coracora, sur de Ayacucho, 1921) cursó estudios en la Universidad Nacional Mayor de San Marcos y en la Escuela Práctica de Altos Estudios, Universidad de París, Francia.

En San Marcos fue Profesor Principal y Director del Departamento de Antropología (1947-1969). Ejerció la Presidencia del XXXIX Congreso Internacional de Americanistas realizado en Lima, 1970. El año 1972 fue declarado Profesor Emérito de la Universidad Nacional Mayor de San Marcos por sus altos méritos académicos.

Cumplió tareas sociopolíticas de Asesor de Gobiernos del Perú los años 1963 y 1988-1989, donde planteó sus ideas social-progresistas destinadas a vencer y superar los obstáculos políticos acumulados a lo largo de centurias en el país.

En México (1989-1995) fue Director del Instituto Indigenista Interamericano. También fue llamado para ser asesor de UNICEF-MÉXICO para plantear estrategias político-sociales de lucha contra la 
pobreza de familias en las áreas desérticas mexicanas (1995-1997) y además asesoró a CONAZA (Comisión Nacional de Zonas Áridas) de México, así como participó activamente en IDISA (Ingeniería para el Desarrollo Integral) en la sierra de Tamaulipas, México.

Entre 1999-2000 ejerció de Consultor del Centro de Investigación en Geografía y Geomática "Ingeniero Jorge L. Tamayo", Secretaría de Educación Pública -Consejo Nacional de Ciencia y Tecnología SEP/CONACYT- México. También ejerció como Consultor del Instituto Panamericano de Geografía e Historia (IPGH), México, 1999-2003.

Como docente internacional fue profesor del "Seminario de Estudios Interdisciplinarios" para la Licenciatura en Estudios Latinoamericanos de la Facultad de Filosofía y Letras, Universidad Nacional Autónoma de México (UNAM), México, 2003-2008.

De regreso al Perú continuó con las investigaciones sociales- antropológicas y además ejerció como Director del Centro de Investigaciones de la Universidad Particular Ricardo Palma, Lima, 20132015.

El 5 de agosto del 2015, fue condecorado con la Orden del Sol por el Gobierno del Perú, reacción oficial tardía, pues el 7 de agosto el maestro José Matos Mar pasó al mundo paralelo del Hananpacha, donde la vida andina, de finita se convierte a transfinita.

\section{NACIMIENTO DE LA ANTROPOLOGÍA PROFESIONAL en San Marcos y el Perú.}

Los años de 1940 a 1960 fueron dos décadas importantes para vertebrar la antropología en el Perú. Es verdad que la antropología peruana surgió sobre los fundamentos metodológicos de la antropología y etnología norteamericanas y bases teóricas relacionadas con el funcionalismo, el difusionismo y el culturalismo, corrientes provenientes de fuente positivista. Se planteaba estudiar el concepto de cultura derivada de las determinaciones utilizadas por el hemisferio norte en el marco de cambios sociales y culturales para conocer las características de las sociedades llamadas "primitivas", "indígenas" o "nativas", destinadas a ser incorporadas a occidente mediante los procesos de aculturación y asimilación de imposición dominante.

La Universidad Nacional Mayor de San Marcos, en proceso de renovación y mejoramiento académico por aquellos años, institucionaliza los estudios antropológicos peruanos al fundar en $1946 \mathrm{el}$ Instituto de Etnología, cuando el Perú transcurría sus días en pleno proceso de transculturación y el movimiento indigenista (1920-1945) rechazaba que el hombre andino fuera incorporado a la civilización occidental, porque habría sido reconocer que no hubo una civilización andina en Sudamérica. El Dr. Luis E. Valcárcel, fundador e introductor de los estudios etnológicos en el país, pensaba que la Etnología sería la ciencia que investigaría la realidad antropológica y social del hombre del ande y su vasta cultura. Este empeño lo llevaría también a fundar en 1956 el Departamento de Antropología en San Marcos, propendiendo a la profesionalización antropológica de los peruanos, en cuyo proceso el Dr. José Matos Mar tuvo acertadísimo trabajo de docencia universitaria, investigaciones, estudios centrados en las comunidades andinas, alienta la educación del campesinado, valora los conocimientos y cultura ancestrales y activa en los medios limeños la información científica acerca del alto nivel sociocultural andinos mediante publicaciones explicativas de raigambre telúrica. 
Los profesionales extranjeros fueron pocos, pero son; desde 1937 Bernard Mishkin estaba estudiando las comunidades llamada de Quispicanchis (Cusco); Harry Tschopik hacia 1940 en compañía de su esposa Marion Tschopik efectuaban investigaciones antropológicas en el altiplano; en 1940 arribó al país Paul Fejos para hacer estudios entre los Yagua amazónicos, luego entre los Bora y después Witoto; John P. Gillin del Smithsonian Institution en 1944 se encontraba trabajando en Moche y publicó sus resultados. Esta actividad antropológica-etnológica propulsó la importancia de la antropología para penetrar en los estudios de la vida comunal, las supervivencias culturales y la vida andina en general. Los estímulos generados por los investigadores extranjeros orientaron a nuestros científicos sociales para estudiarnos, conocer los problemas para superar las condiciones de las comunidades altoandinas y propender los cambios hacia el mejoramiento de los hombres y mujeres andinos.

\section{Al RESCATE DEL HOMBRE Y LA CULTURA ANDiNA.}

El año 1948 el Dr. José Matos Mar es uno de los graduados con una tesis resultado de trabajos de campo en la cuenca del río Cañete (Tupe: una comunidad del área cultural del Kauki en el Perú) y después su doctorado el año 1958 con la Tesis "La Estructura Económica de una Comunidad Andina: Taquile, una isla del Lago Titicaca". El Dr. Matos Mar ingresa a la docencia universitaria e inicia una sucesión de estudios de campo, participación en proyectos, eventos académicos y publicaciones de la especialidad. Así, lo encontramos en el Proyecto Huarochirí en 1955 (Matos Mar et al, 1959). La ex - Corporación Nacional de Vivienda auspicia el importante Proyecto Estudios de Barriadas dirigido por el Dr. José Matos Mar; conformaron el proyecto además el arquitecto inglés John Turner, alumnos del Instituto de Etnología y Arqueología de San Marcos por convenio interinstitucional. Por este tiempo (1950-1960) estaban ejecutándose proyectos en diversas partes del Perú, tales como: el Proyecto Perú-Cornell de Antropología Aplicada, el Proyecto Tambopata como parte del Programa de las Naciones Unidas y Órganos Especializados de esta entidad internacional: UNESCO, UNICEFF, FAO, OMS, OIT, etc., para estudiar comunidades indígenas del Ecuador, Bolivia y Perú; como resultado de tales actividades y luego de la creación del Departamento de Antropología en San Marcos por el Dr. Valcárcel (1956), el país contaba con un buen número de científicos sociales trabajando por el Perú Andino como investigadores y docentes universitarios: Luis E. Valcárcel, Jorge C. Muelle, Pedro Weiss, Pedro E. Villar Córdova, José Matos Mar, José Mejía Valera, Aníbal Ismodes Cairo, José María Arguedas, Gabriel Escobar, Julio Cotler, Héctor Martínez, Mario Vásquez, Emilio Mendizábal, Aníbal Quijano, Carlos Delgado, Federico Kauffmann, Stefano Varese, Luis Lumbreras, Rosa Fung Pineda, Duccio Bonavia, Ramiro Matos Mendieta, etc.

El Dr. Matos Mar venía trabajando en el Proyecto Lago Titicaca a mediados del siglo XX, el cual comportaba estudios pluridisciplinarios, pues se contaba con la presencia del doctor Jehan Vellard (médico francés), Francois Bourricaud (sociólogo francés) y alumnos del Instituto de Etnología de la Universidad de San Marcos donde colaboraron el Museo de la Cultura Peruana, el Instituto Francés de Estudios Andinos y la Universidad de San Andrés (Bolivia). Como resultado del proyecto se publicaron muchos artículos en la Revista del Instituto Francés de Estudios Andinos y en la Revista del Museo Nacional por los profesionales ejecutores de los trabajos de campo. En este Proyecto fueron estudiadas las poblaciones de las riberas del lago, las islas de Taquile, Amantaní, la isla Soto, la Península de Capachica, así como las islas del Sol y La Luna en el lado boliviano. Durante estas investigaciones, el Dr. Matos Mar siempre ha propiciado la presencia activa de estudiantes no sólo de Etnología/Antropología/Arqueología, sino también de Biología (estudio de plantas autóctonas) y zoólogos (estudio de los animales oriundos), adelantándose a la multidisciplinaridad contemporánea por muchas décadas. 
El mismo Dr. Matos Mar consideró importante y de primer orden el trabajo de gabinete, catalogación archivística, documentos etnológicos del Archivo Arzobispal, informes de curatos y visitas de los siglos XVI, XVII y XVIII con la colaboración de Julio Basto Girón, Aníbal Quijano, Teófilo Espejo Núñez, Dora Rodríguez, Julio Cotler y Aída Vadillo, todos connotados estudiosos peruanos. También cuentan en este trabajo de información un fichero bibliográfico sobre el Perú Antiguo, el fichero de etnología andina con más de 10,000 títulos y recopilación de las fuentes primarias de los cronistas, muy escasas en aquellos años.

Unas de las mayores contribuciones del Dr. Matos Mar es la de haber sido gestor y organizador del Instituto de Estudios Peruanos (IEP). Algunos autores de la década 1960-1970 señalan que fue el verdadero fundador de este Instituto, el cual, como muy pocas instituciones no estatales, ha contribuido tanto a la maduración metodológica y teórica de las ciencias sociales peruanas, pero en mayor profundidad al conocimiento acerca de la estructura de relaciones sociales y culturales y los estímulos sociohistóricos para los cambios, movimientos sociales, migraciones plurirregionales y traslaciones complejas de valores, etnomitologías, costumbres, ideas etnorregionales, comportamientos y conductas andinoamazónicas a las ciudades de la costa y especialmente a Lima, cuyos procesos de dinámica social convergente la hicieron transformarse en la metrópoli de todas las sangres que es hoy.

Esta amplia y compleja temática tiene como principal promotor, investigador, autor y ejecutor al Dr. Matos Mar, pues ya sea en las instituciones estatales como en las particulares, ha demostrado ser un intelectual sanmarquino a cabalidad y un hombre profundamente compenetrado con su trabajo profesional y de amor al Perú.

Las publicaciones institucionales, personal, colectivas o en coedición tienen el sello y orientación del estilo claro, directo y realista del Dr. Matos Mar; son ejemplos: Breve Introducción al estudio de la Realidad Nacional, 1963, Facultad de Educación, Departamento de Orientación y Asistencia Social, Lima; fueron coautores Romeo Luna Victoria S.J. y José Antonio Encinas Pando en el aspecto económico; el análisis social estuvo a cargo de José Matos Mar; las relaciones políticas fueron enfocadas por Francisco Miró Quesada, Andrés Townsend Ezcurra y Jorge Bravo Bressani; la educación fue tratada por Emilio Barrantes y la cultura estuvo confiada a Sebastián Salazar Bondy. Se analizó la realidad nacional de los comienzos de la década 1960-1970 en visión y panorama histórico-social en el contexto de las relaciones internacionales, sin cuyas perspectivas no habría sido posible explicarla. En el libro Estudios sobre la Cultura Actual del Perú, publicado por la UNMSM, Lima, 1964, compila publicaciones variadas de etnólogos peruanos y extranjeros de primera línea, enfocando los procesos de cambios sociales y culturales del Perú. El volumen de gran calidad incluye temas del Indigenismo en el Perú por Luis E. Valcárcel; La Estratificación Social en el Callejón de Huaylas de William P. Mangin; Louis Faron informa sobre la Formación de dos comunidades Indígenas en un Valle de la Costa Peruana; La Propiedad en la Isla de Taquile es tratada por José Matos Mar; Bernard Mishkin escribe sobre posesión de la tierra en la comunidad de Kauri, Quispicanchis; Gabriel Escobar informa sobre Sicaya, una comunidad mestiza de la sierra central del Perú; Puquio, una cultura en proceso de cambio es analizada por José María Argüedas; el cusqueño Oscar Núñez del Prado informa acerca de El Hombre y la Familia: su Matrimonio y Organización Político Social en Q'ero y los clubes de provincianos en Lima es informado por William Mangin. Magnífico volumen que abarca casi todo el Perú.

En 1970 Matos Mar publica un extraordinario artículo en la Rev. Copé, Vol. I, Número 2, titulado: "Los procesos de desarrollo histórico de la Sociedad Peruana", en el cual señala con mucho énfasis los factores que dominan al mundo andino y los desarrollos desiguales, pero también señala nuevas perspectivas y su pujante continuidad social y cultural en el Perú de todos los tiempos. 
En la serie Perú Problema el Dr. José Matos Mar abarca diversos tópicos de su especialidad relacionada siempre con la población andina: Hacienda, comunidad y campesinado en el Perú. La dicotomía hacienda/campesinado, es en realidad hacienda/comunidad, debido a la comprensión de que representan la pervivencia de una organización en la cual convergieron la tradición andina e hispana. Considera que las comunidades andinas tienen los conocimientos y la vitalidad para ser aprovechados en la formulación de los planes de desarrollo económico y social, ampliación del mercado interno y la consiguiente industrialización. En otras palabras, el Dr. Matos Mar contradice las opiniones de la burguesía peruana, negativa y denigrante sobre los hombres y mujeres andinos; cambia tal opinión y la transforma al descubrir, mediante los estudios de campo, que se trata de poblaciones históricamente dominadas y maltratadas, pero que ahora presentan procesos de cambios y que están destinadas a ser quienes transformen la realidad peruana hacia la nación autónoma contemporánea.

El trabajo titulado "El Pluralismo y la Dominación en la sociedad Peruana. Una Perspectiva Configuracional”, publicado en el volumen Dominación y Cambio en el Perú Rural, demuestra que la tierra andina es un contexto natural con fuerte carácter simbólico y repositorio esencial de nuestra territorialidad como nación oriunda de larga data y que algunas obras arqueológicas -como los andenesestablecen la comunidad "tierra/hombre", constituyéndose en fundamentos de relación metafórica con los asentamientos andino-sociales vivos. Al dirigir el Proyecto del Valle de Chancay el Dr. Matos descubre la dicotomía del cambio y de la modernidad en el campo, lo cual implica la aceleración transformativa de la antigua comunidad en los Andes.

El Dr. Matos Mar en sus clases y estudios ha recuperado la configuración "tierra/hombre" de carácter netamente andino -por ejemplo el ayllu: sistema de relaciones estructurales entre el hombre y la tierra- porque el manejo andino de la tierra funciona como unidad etnosocial. Nuestro autor en sus obras antropológicas establece que la comunidad "tierra/hombre" se nutre permanentemente de la territorialidad social, porque en las relaciones con la tierra de los Andes se fortalece la organización social, donde hombres, mujeres y niños están interrelacionados familiarmente al intercambiar recíprocamente productos de la tierra, ganado, servicios y lealtades intraculturales antiguas y actuales.

Matos Mar considera que la transformación geomórfica de los Andes por medio del trabajo social para la agricultura andina es un verdadero sentimiento comunitario, fraternal, socialmente indiviso y respetuosamente ancestral, pues los andinos practican ideas telúricas de diferentes relaciones con la tierra referidas a que en el seno de ésta alberga la asunción de continuidad biológica y social de sus ancestros, enlace vital que protege su familia, alimentación y les revela la cosmografía telúrica, cuyas formas visuales sugieren relaciones complejas inmateriales evocadoras de los apus, extendidas a la enhebración del pensamiento andino de todos los tiempos con el orden cosmográfico, vertiente significativa hacia su integridad sociohistórica. Por eso, el Dr. Matos Mar retrotrae al ayllu desde la antigüedad arqueológica, que es un vocablo oriundo, significador del territorio antiguo y actual integrando tierras labrantías, espacios cosmográficos, aquellos altos apus glaciares, las áreas de andenes, quebradas, aguas, flora y fauna como factor colectivo de pertenencia sociocultural. Los espacios cosmográficos altos, bajos y altipampas multiplican las plantas cultivadas y simbolizan el ordenamiento del territorio y su transformación de lo simple a lo complejo, alcanzando la mitificación eidética del campesino, junto con los productos de la tierra, los cuales son la trama de su vida.

Así, el pensamiento antropológico del doctor José Matos Mar continúa contextualizado a los Andes al unísono de los tambores, quenas, pututos, sikus y zampoñas del evolutivo mundo andino; a la dinámica y conjuro andino él ha respondido con un novísimo gran libro titulado: Perú. Estado Desbordado y Sociedad Nacional Emergente; Centro de Investigación, URP, Lima, 2012. En el volumen de 573 
pp. Matos Mar identifica y define al Perú Oficial capitalista con fuertes resabios neocolonialistas practicantes del neoliberalismo ideológico político-económico, frente al Otro Perú, dinámico y cambiante para rebasar al Estado y ponerlo en crisis, comprobando su acierto del Desborde Popular y Crisis de Estado del año 1984, cuya publicación ya cuenta con 15 ediciones. El libro del año 2012 publicado por la Universidad Ricardo Palma es datacional, testimonial y polémico, pero al mismo tiempo de confianza y esperanza de que el desborde popular siga por la senda de los éxitos, los desbordadores continúen educándose y trabajando para madurar como ciudadanos destinados a conducir al país hacia metas de cultura originaria respetada, progreso técnico-científico y solvencia ciudadana económico-política.

En este libro el Dr. José Mar se identificó con la gente del Perú marginado (los habitantes de las barriadas populares) y tuvo visión antropológico- social para ver y comprender a los migrantes, a quienes depara un papel activo en la transformación sociopolítica y económica del país. Los migrantes trajeron a Lima nuevas fuerzas de trabajo, empujes a pulmón en todos los lugares donde pudieron establecerse, y por lo menos, sacaron de la molicie y el quietismo inútil a los habitantes de la vieja ciudad de Lima.

Los migrantes abrieron nuevos espacios hacia las pampas desérticas, despreciadas por empleados y burócratas citadinos, ampliando las posibilidades para el crecimiento de la ciudad, aunque su desarrollo demoraría de una a dos décadas para ponerse en valor. Pero su "desborde popular" sobrepasa en acción y dinámica poblacional al estancamiento del Estado dominado desde San Isidro, Miraflores, Surco y La Molina, debido a que tradicionalmente menosprecian a las fuerzas sociales andinas.

Mediante la migración lograron romper los moldes formales de la dominación agraria, del dominio político municipal, de la corrupción del foro y del segregacionismo racial, manifestado en la diferenciación social contra los "serranos", "cholos" e "indios", denostaciones que fueron hechas trizas al revitalizar a la vieja y apoltronada Lima, bien analizada y comprendida por el doctor Matos Mar, al dar sentido futuro y confianza ideológica a la gente migrante que rebaza todos los cauces a la dinámica de su docencia cultural. Es evidente que el desbordador de la segunda mitad del siglo XX es el emprendedor de hoy que le muestra al "Estado oficial" su inercia que estanca al país, por eso, exclamamos que no sólo fue promotor sociopolítico, sino un gran renovador social para todos los peruanos explicados en la claridad de sus textos.

Por desgracia para el IEP, no para José Matos Mar, los graduados de la PUCP invadieron silenciosamente al IEP, y en cargamontón, lo defenestraron. Desde entonces, el IEP no ha vuelto a estudiar la problemática andina nacional, deslizándose al elitismo, el individualismo y la exquisitez señorial con olor a naftalina occidental.

En homenaje al Dr. José Matos Mar en vida (2013), el XVII Taller de Investigaciones Sociales de la Facultad de Ciencias Sociales de la Universidad Nacional Mayor de San Marcos se denominó con el epónimo nombre del Dr. José Matos Mar, eminente antropólogo peruano y uno de los fundadores de las Ciencias Antropológicas en el Perú, al cual le damos las gracias por todos los beneficios académicos que hemos recibido a través de sus brillantes clases en aula y su capacidad crítica y científica, verdaderamente desbordantes. 


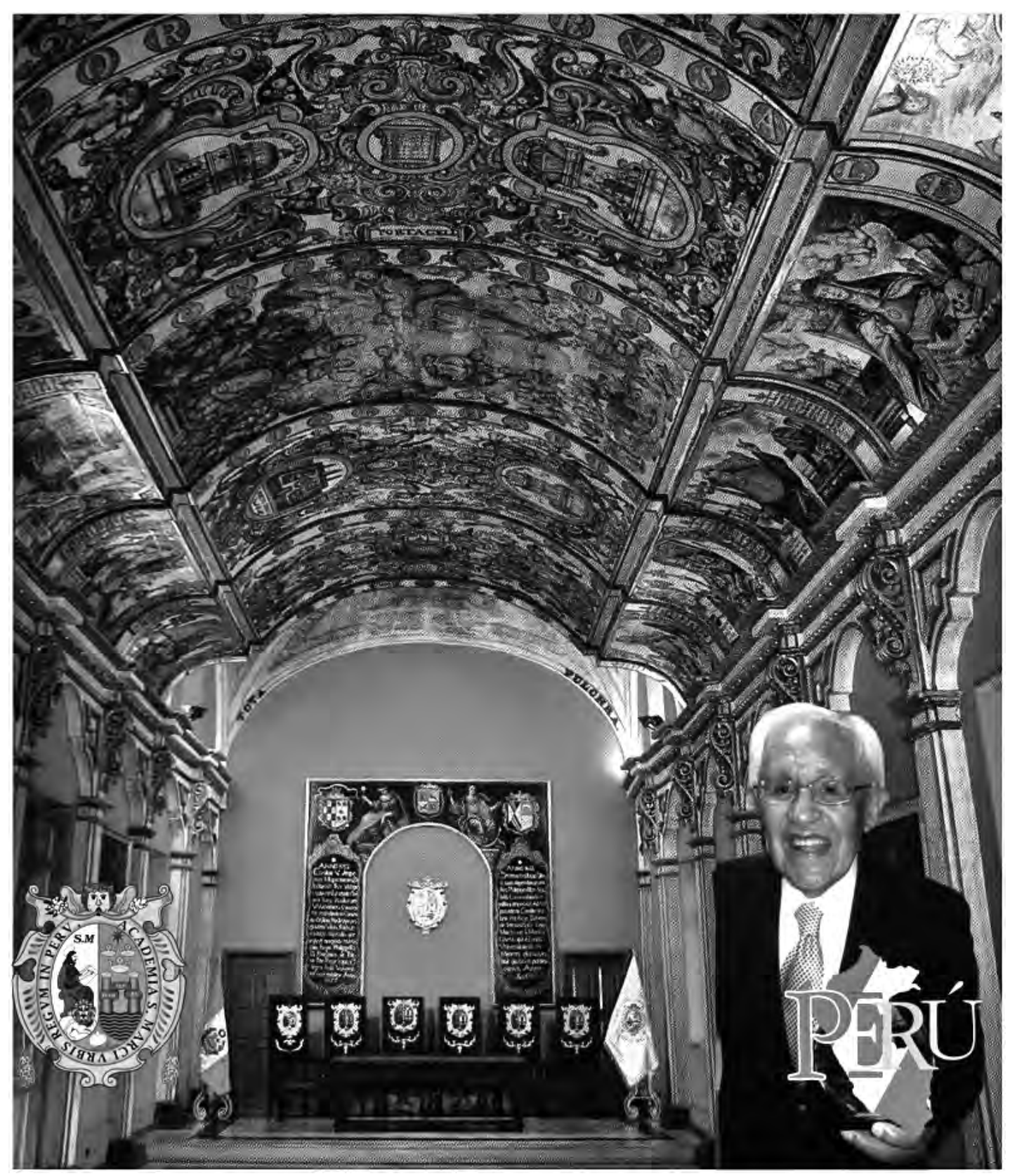

El Dr. José Matos Mar en el marco de los símbolos emblemáticos de San Marcos, en razón de su magisterio docente sanmarquino. 
